\title{
Track-Before-Detect Filter Banks for Noise Object Tracking
}

\author{
Przemysław Mazurek
}

\begin{abstract}
The Track-Before-Detect (TBD) filter banks is proposed for the processing of noise object that are additive to the background noise. Spatio-Temporal TBD algorithm uses the preprocessing of measurement. The modified moving standard deviation filter is applied. The correction of the results for the selection of the highest possible filter banks window is proposed. Position and velocity errors are evaluated numerically for two smoothing coefficients. Monte Carlo test shows that all filter banks allow the tracking if the standard deviation of the background is below 1.3.
\end{abstract}

Keywords-Track-Before-Detect, tracking, estimation, noise object

\section{INTRODUCTION}

$\mathbf{T}$ HE tracking algorithms are very important in numerous applications. The air, space, water surface, underwater surveillance applications are important for example [1], [2], [3]. The tracking systems are very sophisticated. The main parts of systems are related to the detection, tracking and assignment. Most systems are related to the multiple objects tracking, so all parts of the signal processing parts need careful design and implementation. Typical tracking systems are based on the mentioned detection and tracking scheme [4], [5], [1] (Fig. 1).

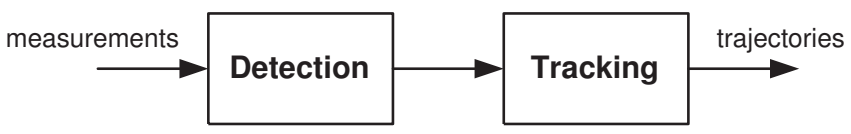

Fig. 1. Conventional tracking scheme.

The detection is based on the threshold algorithm (fixed or adaptive threshold level). The detected positions are processed by the tracking filters that are used for the signal-to-noise ratio (SNR) improvement. The noise measurement gives quite low SNR values so multiple false detections and missed detection occur. Tracking filters use the knowledge about the motion model so reduction of the influence of the noise could be obtained. The assignment algorithm is applied to the tracks maintenance. The observation should be assigned to the appropriate track, new tracks should be created if a new object is in the range and the removal of the tracks for objects that are outside of the range is necessary.

Conventional approach fails if the noise level is high and the signal values related to the object are low (Fig. 2). The

This work is supported by the UE EFRR ZPORR project Z/2.32/I/1.3.1/267/05 "Szczecin University of Technology - Research and Education Center of Modern Multimedia Technologies" (Poland).

P. Mazurek is with the Department of Signal Processing and Multimedia Engineering, West Pomeranian University of Technology, 26-Kwietnia 10 71-126 Szczecin, Poland (e-mail: przemyslaw.mazurek@zut.edu.pl). object is hidden inside the noise floor and the tracking is not possible.
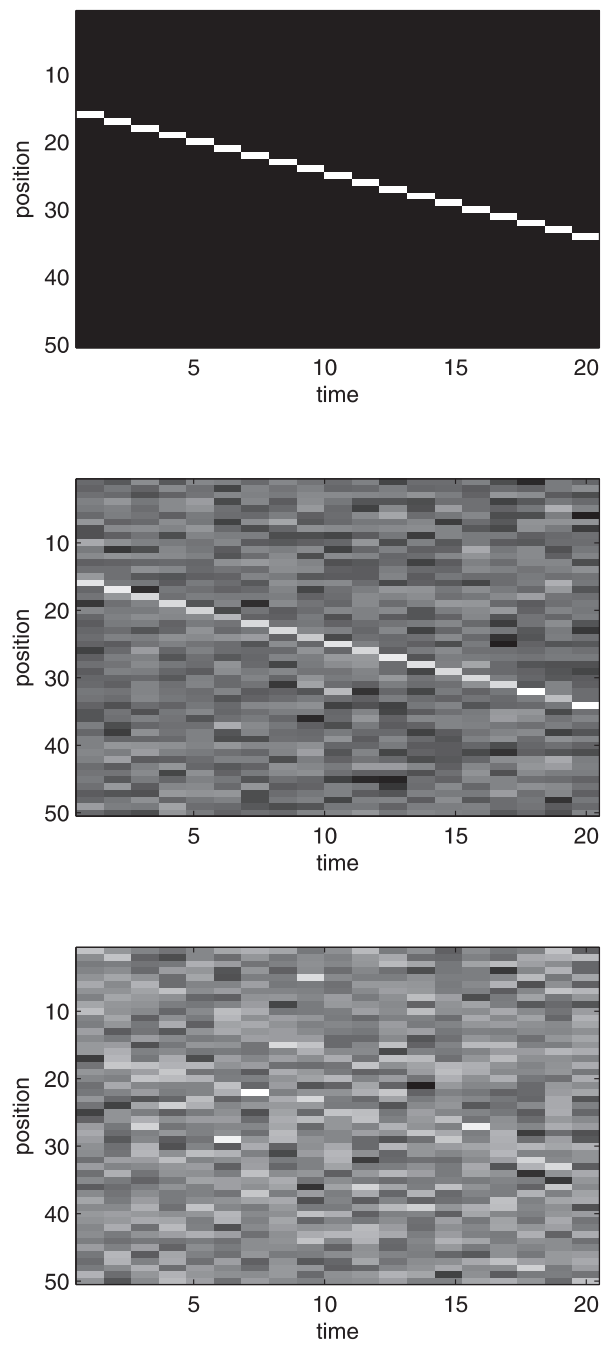

Fig. 2. Example of 1D measurements: Simple to detect object; Measurements disturbed by the Gaussian background noise; Object hidden in the Gaussian background noise.

Alternative approach, based on the opposite scheme is necessary. The track-before-detect (TBD) approach (Fig. 3) allows the tracking of such objects, even if $S N R<<1$ [1], [6], [7]. The number of computation is huge, because all hypothetical trajectories should be verified, even if there is no object in the range [8], [9]. The cost of conventional approach is linear function of the number of observed object, quite often. Accumulative approach applied in TBD algorithm allows the filtering of the signal related to the every trajectory [6]. 


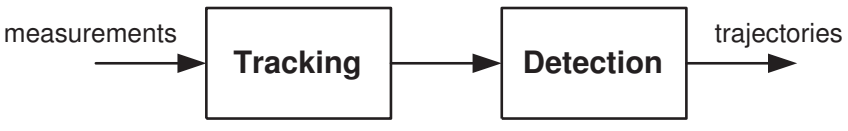

Fig. 3. Track-Before-Detect scheme.

TBD systems could be applied for all kinds of signals that are used in surveillance applications. Radar and infrared signals could be processed using directly or after additional preprocessing. Data fusion, from the same type sensor is possible. Multiple sensors are important for the estimation of the proper state of the object. Radar measurements are sensitive to distance especially, but the angular resolution is low. Infrared measurements give the superior resolution of bearing but the distance measurements are not possible using single sensor. Application of multiple sensors located at different position allows solving problems of the single sensor.

Alternative option is based on the data fusion from two different sensor types located at the same or different locations. Combined measurements using radar and infrared sensors allow estimation of the object state using data fusion [7].

\section{Spatio-Temporal Track-Before-Detect ALGORITHM}

The Spatio-Temporal TBD algorithm is recursive algorithm for multidimensional signal processing [10], [11], [9], [12]. The formula (1a) is the initialization of the state space. The formula (1b) is the motion update responsible for the prediction and noise suppression. The formula (1c) is the information update responsible for the balance between new measurement and predicted values.

\section{Start}

// Initial:

$$
P(k=0, s)=0
$$

For $k \geq 1$ and $s \in S$

// Motion Update:

$$
P^{-}(k, s)=\int_{S} q_{k}\left(s \mid s_{k-1}\right) P\left(k-1, s_{k-1}\right) d s_{k-1}
$$

// Information Update:

$$
P(k, s)=\alpha P^{-}(k, s)+(1-\alpha) X(k)
$$

\section{EndFor}

End

$\begin{array}{cll}\text { where: } & & \\ S & - & \text { state space } \\ s & - & \text { state (spatial and velocity components) } \\ k & - & \text { time moment } \\ \alpha & - & \text { smoothing coefficient } \alpha \in(0,1) \\ X(k) & - & \text { measurements } \\ P(k, s) & - & \text { estimated value of objects } \\ P^{-}(k, s) & - & \text { predicted value of objects } \\ q_{k}\left(s \mid s_{k-1}\right) & - & \text { state transitions (Markov matrix) }\end{array}$

This algorithm allows the suppression of the zero valued noise and enhancement of positive or negative signals.

Low-pass filtering property is related to smoothing coefficient and Markov matrix. High $\alpha$ value gives narrower lowpass filtering, so more steps $(k)$ are necessary for steadystate. Such conditions influence the improving SNR and allow detection of weaker objects.

The Markov matrix is responsible for the switching between trajectories. High ratio of switching is the source of SNR reduction. Trajectories that are fitted to the expected trajectories of object are desired. This matrix is very sparse and ST TBD algorithm should be not implemented using (1b) formula directly.

The output of ST TBD algorithm is the formula (1b) or (1c). The predicted state-space (1b) is assumed as an output in this paper. The state space influences the trajectories definition and overall quality of tracking. Simplest variant, based on the direct relation between measurement and state-space, is assumed. The 1D measurement are extended to the 2D state space, where the second dimension is the velocity. Such formulation for lack of the switching between trajectories is the velocity filter. It allows the testing of the performance for ST TBD algorithm for best case - maximal fitting of trajectories. Markov matrix has zeros and ones only. Allowing of switching between trajectories reduces overall performance, so boundary case is tested in this paper.

\section{TRACKING OF NoISE OBJECT}

The noise object is special case of signal - the mean value is zero and values are random (Fig. 4). Such signal cannot be processed by ST TBD algorithm directly. The stealth technologies are quite often applied to some objects, so weak signals are observed. The stealth techniques are applied for the reduction of periodic signals that are common for engines. Periodic signals could be detected using frequency analysis, that is the main technique for the detection of such objects. Objects could be sources of signal, but could be also a barrier for the background noise.

The noise difference between object and the background could be applied for the tracking of such objects. Low differences between object and the background cannot be observed, unfortunately. Long time measurements and special TBD algorithms allow the detection and tracking.

The application of the variance/standard deviation estimator for the preprocessing of measurements (Fig. 5) is proposed in [12]. Fixed size of the object (number of samples) and the fixed size of the moving window are applied.

$$
X(k, s)=\operatorname{std}(M(k, s-L) \cdots M(k, s+L))
$$

The measurements $M$ are preprocessed using window size $2 L+1$. Another approach is based on the comparison of two distributions. The global distribution is calculated for overall measurements for the current time moment. Sliding window is applied for the calculation of the local distribution. Both distributions are compared for every position of the moving window. Comparison is possible using chi-square formula for example [12] or dot product formula [13]. 

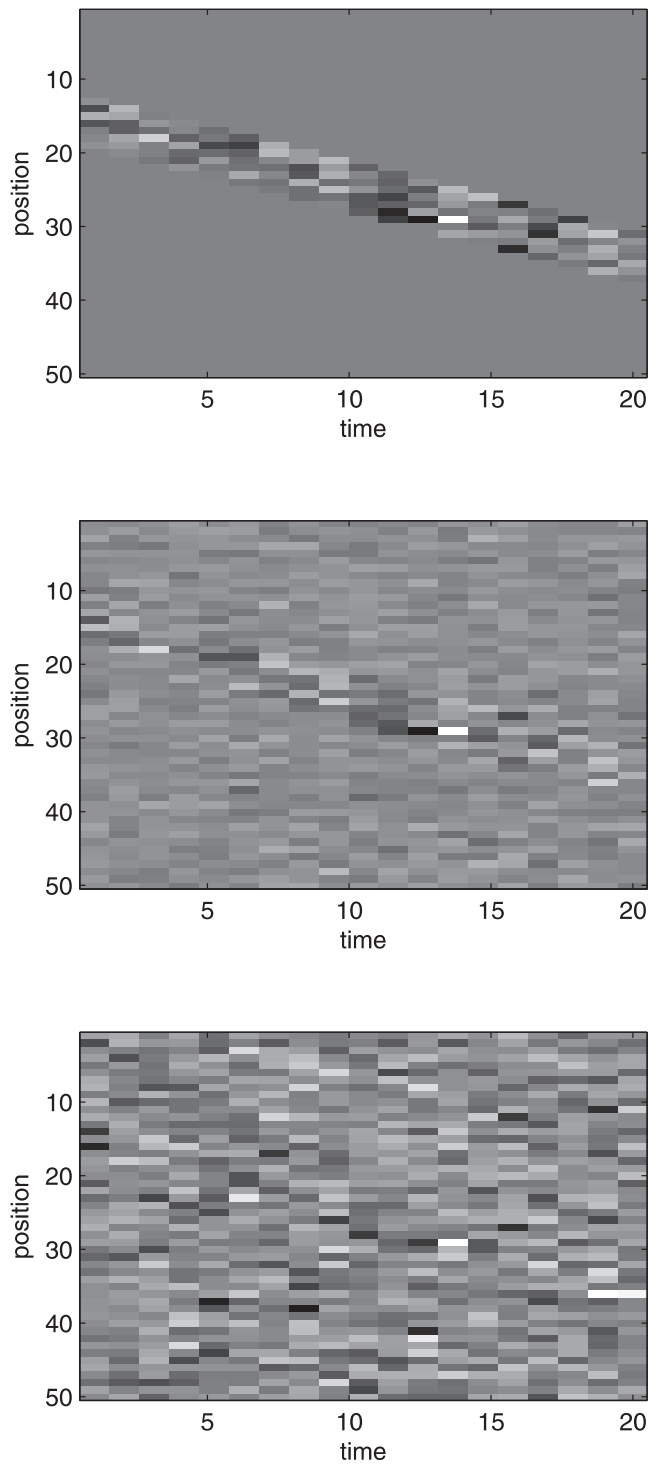

Fig. 4. Example of 1D measurements of noise object: Simple to detect object; Measurements disturbed by the Gaussian background noise; Object hidden in the Gaussian background noise.

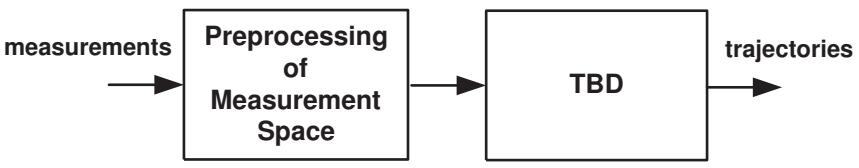

Fig. 5. Preprocessing of measurement space scheme.

\section{FILTER BANK FOR TBD}

The standard deviation is an interesting estimator that could be applied for Gaussian background noise and the additive object Gaussian noise signals separation. The size of the object could be variable, so detection should be based on the variable window size.

The computation of the standard deviation is based on the computation of the local mean value. The correction constant is necessary for the comparison of standard deviations for different number of samples. The standard deviation is related to the radius of hypersphere, where the number of samples is the dimension. The smaller number of samples is related to the subset of the hypersphere in lower dimension, with the same radius. The most important problem is that signal is random. The computation of the standard deviation is based on the local mean value, so subset has not identical mean value. It is a result of the radius change.

The proposed technique is based on the computation of the mean value for higher dimension (longest window size or all samples). The mean value is applied for the computations of standard deviation for the smaller sample sets. It is also computationally important. Instead formula (4), new proposed formula is applied:

$$
X_{\text {bank }}(k, s)=k_{\text {bank }} \sqrt{\frac{1}{2 L} \sum_{i=-L}^{L}(E-M(k, s+i))^{2}}
$$

The $k_{\text {bank }}$ correction constant is the value dependent on the filter (Fig. 6). The number of filters should be reduced due to high computation cost of TBD.

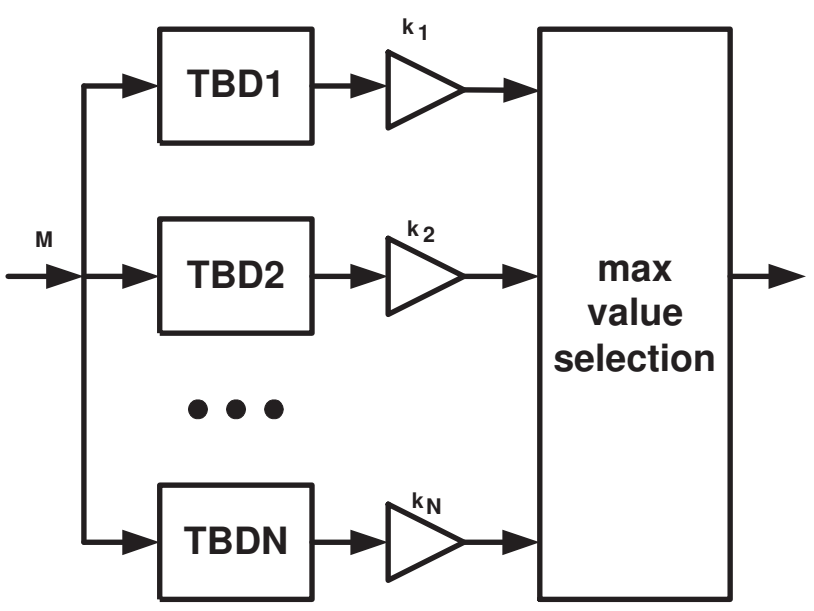

Fig. 6. Filter banks schematic.

The value $E$ is the mean value for all samples, not only inside the moving window. Each filter is assigned to the different window size so longer window size filter should be preferred. The following relation should be applied:

$$
k_{1}<k_{2}<\cdots<k_{N}
$$

for example:

$$
k_{i}=2 L+1
$$

as a weight value dependent on the window size. The ST TBD is linear transformation of signal, so correction constant could be added not to the input measurement transformation, but for TBD output result. The selection of the maximal value could be used as data fusion operation, but other approaches are possible also.

\section{RESUlts}

The Monte Carlo test is assumed for the performance analysis of TBD filter banks. There are 3000 positions and 11 velocities. The $1 \mathrm{D}$ signal is processed and the standard 


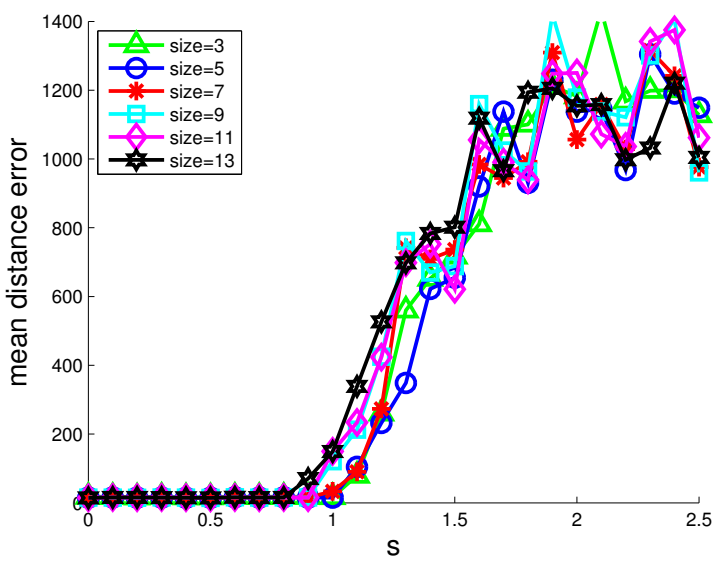

Fig. 7. Mean distance error (Noise object width $=5, \alpha=0.95$ ).

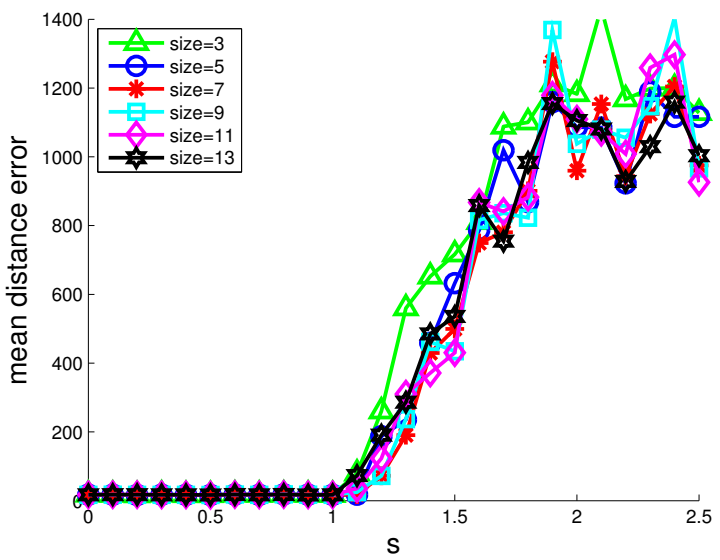

Fig. 8. Mean distance error (Noise object width $=7, \alpha=0.95$ ).

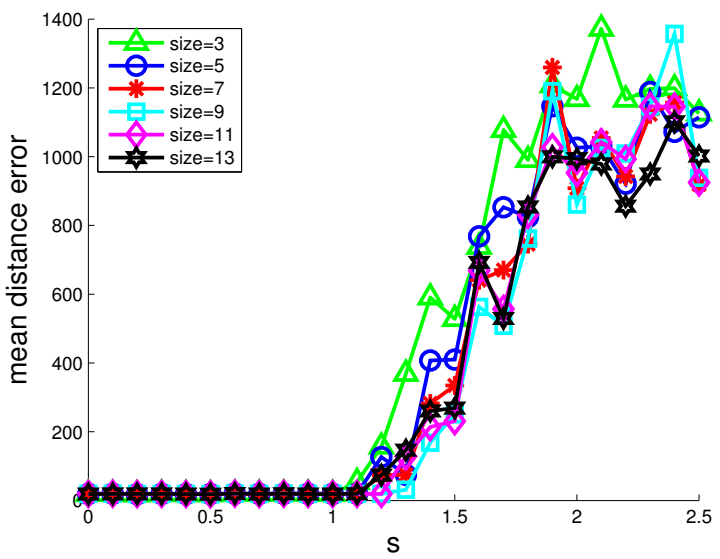

Fig. 9. Mean distance error (Noise object width $=9, \alpha=0.95$ ).

deviation of the object is 1.0 , the standard deviation of the background noise is variable (0-2.5), but fixed for single test.

There are 1000 tests for every tracking scenario (different lengths of the object are assumed: 5, 7, and 9).

Mean distance error is depicted in Figs. 7- 12 after 80 iterations, for $\alpha=0.95$ and $\alpha=0.98$. The estimated position

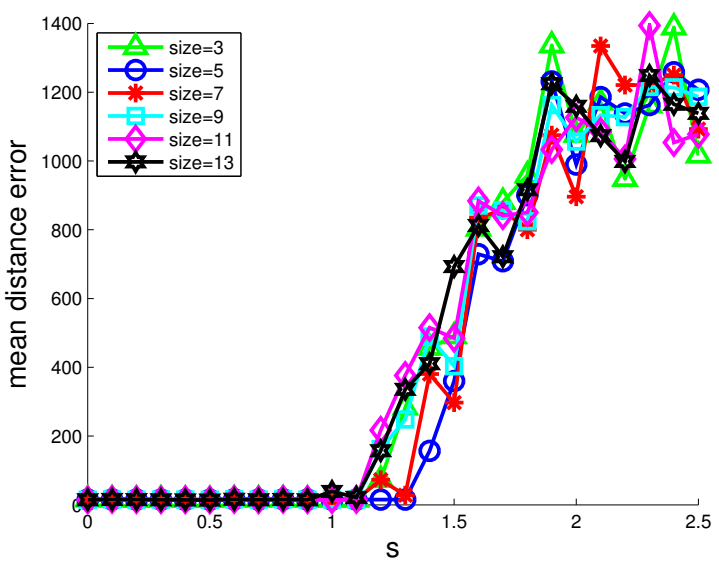

Fig. 10. Mean distance error (Noise object width $=5, \alpha=0.98$ )

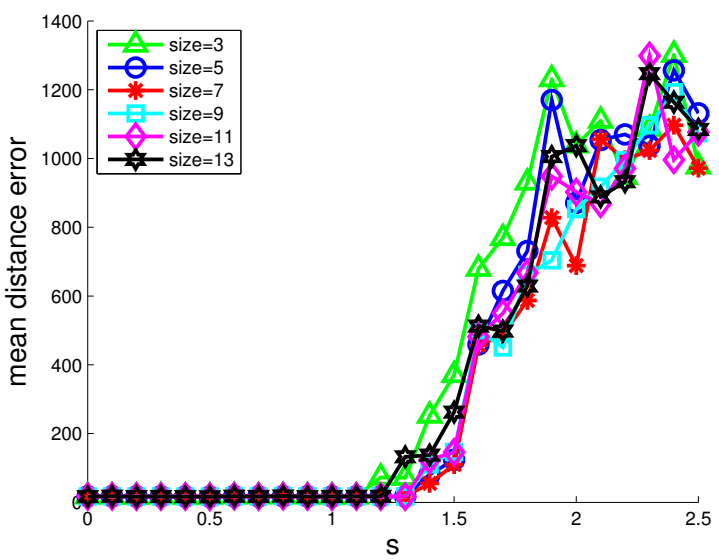

Fig. 11. Mean distance error (Noise object width $=7, \alpha=0.98$ ).

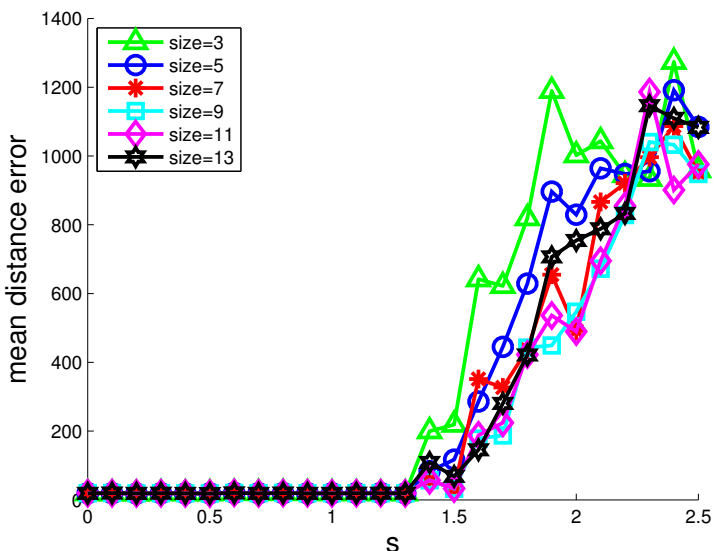

Fig. 12. Mean distance error (Noise object width $=9, \alpha=0.98$ ).

is the obtained as position for largest value of the state space. This is not optimal solution for the real tracking system, but it could be used for tests. Much better results could be obtained if the second tracking algorithm is applied for outputs, for multiple target tracking. 


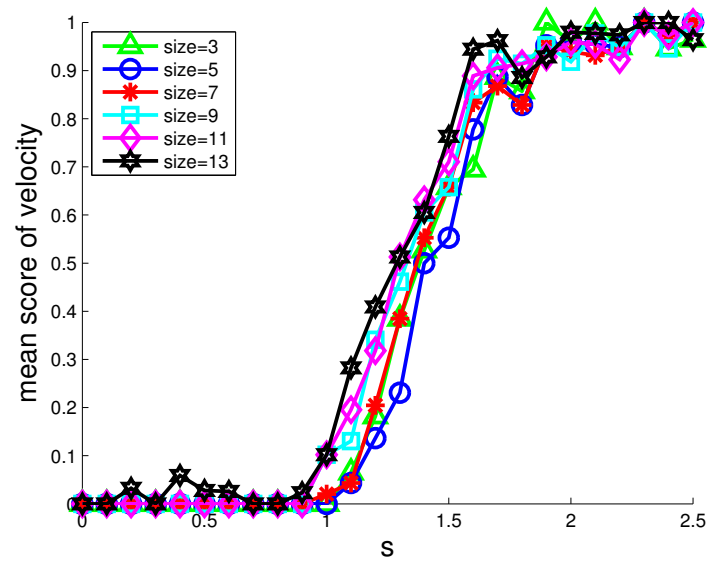

Fig. 13. Velocity error score (Noise object width $=5, \alpha=0.95$ ).

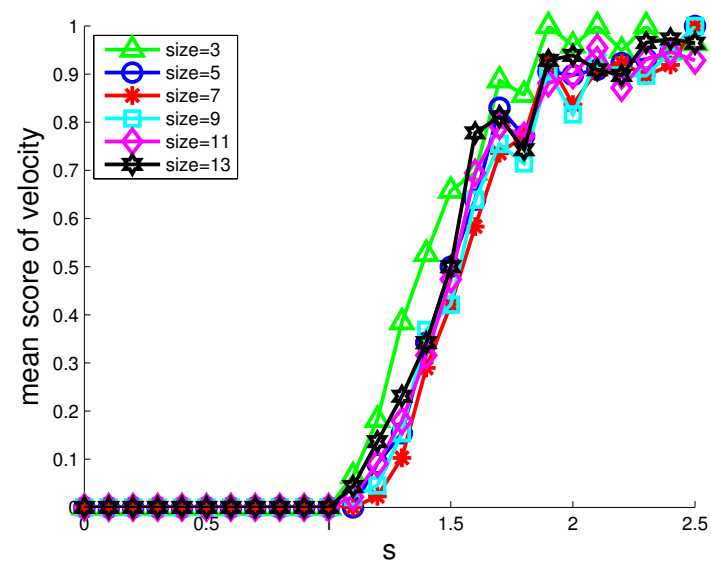

Fig. 14. Velocity error score (Noise object width $=7, \alpha=0.95$ ).

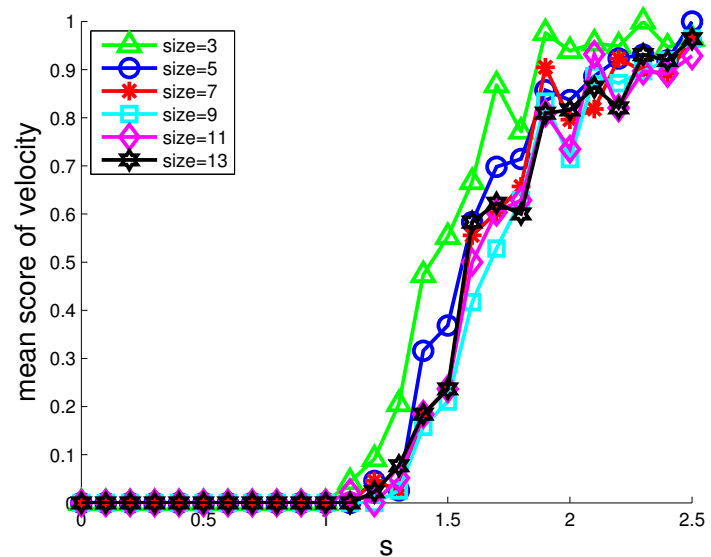

Fig. 15. Velocity error score (Noise object width $=9, \alpha=0.95$ ).

Estimation of the velocity together with the position is also possible. The next results are related to the object velocity error (Fig. 13 - 18). The velocity cannot be considered without the position. The scoring approach is assumed for the velocity and position criteria. The value " 1 " is assigned to the case when the object position error is within $\langle-10+p, p+10\rangle$

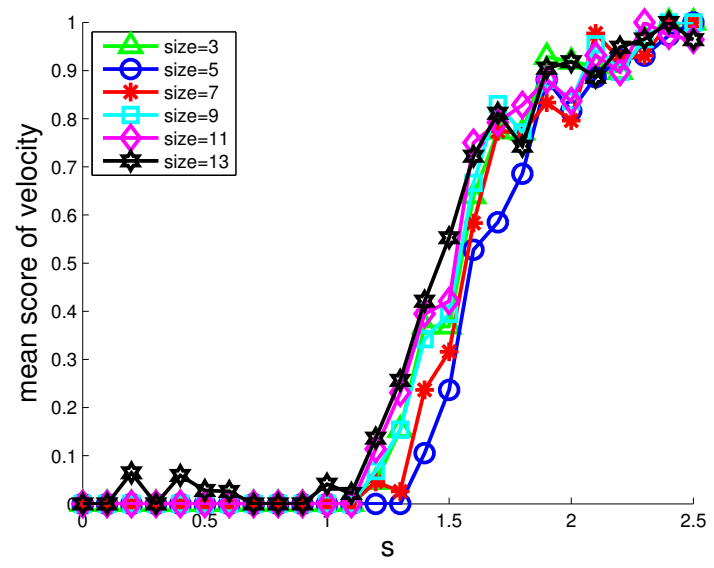

Fig. 16. Velocity error score (Noise object width $=5, \alpha=0.98$ ).

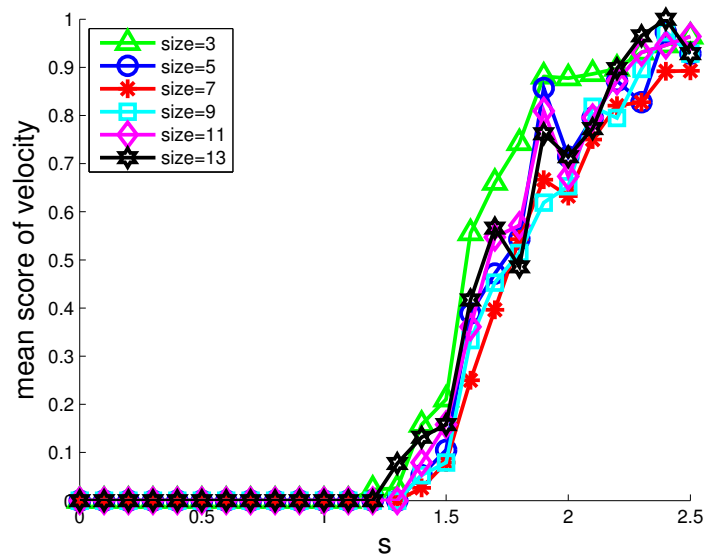

Fig. 17. Velocity error score (Noise object width $=7, \alpha=0.98$ ).

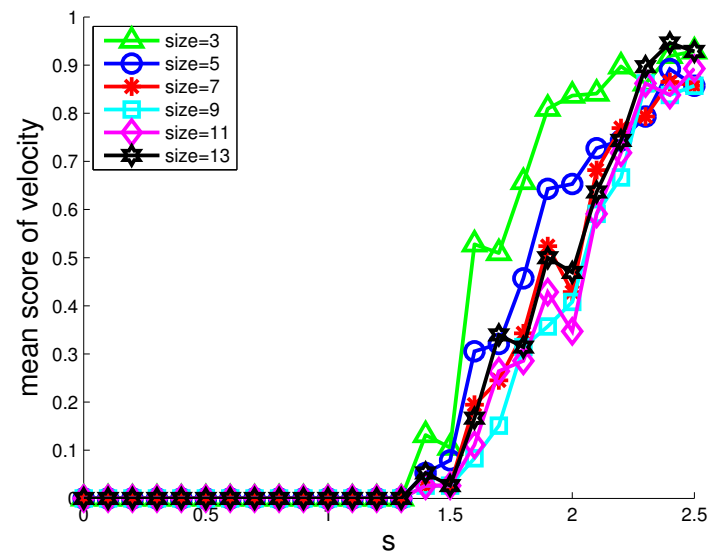

Fig. 18. Velocity error score (Noise object width $=9, \alpha=0.98$ ).

range, where the $p$ is the known position and the velocity is within $\langle-1+V, V+1\rangle$ range, where $p$ is the known velocity.

Data fusion using filter bank according to Fig. 6 is shown in Fig. 19 for $\alpha=0.98$. The selection of the maximal value from multiple TBD gives the best results. 


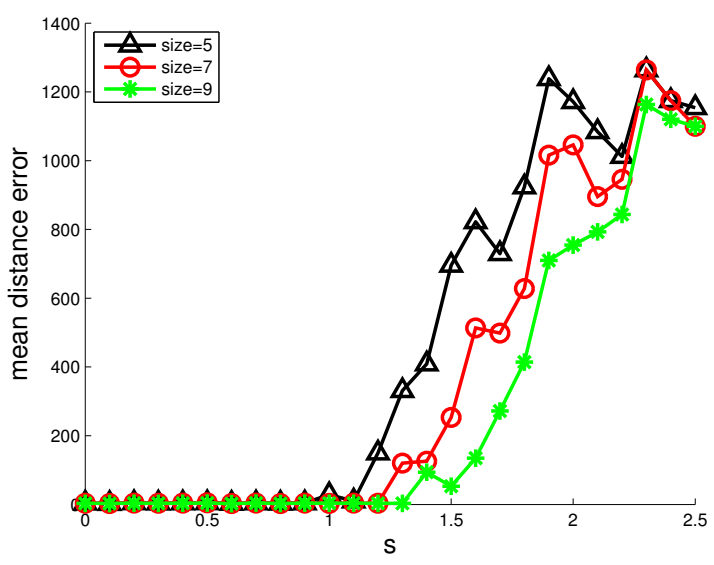

Fig. 19. Mean position error $(\alpha=0.98)$ after data fusion for different object sizes.

\section{Discussion}

The position error is not equal to the zero for Monte Carlo test even for high SNR case. It is a result of the noise type of the object signal. This is not an important problem for the tracking. Such differences are obtained for the single estimation of the position using maximal value. The observation of the results for limited position range using an additional conventional tracking filter is possible. The reduction of the error could be obtained using hierarchical tracking approach.

The increasing of the background noise influences the detection possibilities. The detection is the function of the object size, because larger objects are simpler to detect. The detection of the object with size 5 is possible for $s=1$, with size 7 is possible for $s=1.1$ and with size 9 is possible for $s=1.3(\alpha=0.95)$. The detection of the object for higher background noise is still possible, but the performance is lower, so mean value of distance error is higher. Application of the second tracking filter with gate that is applied for the selection of the testes area should be considered for the further improvement.

The tracking and detection possibilities are improved for the larger smoothing coefficient $(\alpha=0.98)$, for example $s=1.4$ for object size 7 .

The object of the assumed size is well detected by the fitted filter (similar widths of the filter and object), what shows that filter bank approach is desired. The fitted filter results are located in right part of figures. Separate results of the TBD filters are considered for testing this behavior.

The velocity test using proposed technique shows that velocity could be estimated together with position with good accuracy. It shows that position and velocity are related to the object for low background noises. Similar results for the curves are obtained. The fitted filter results are located in right part of figures.

\section{CONCLUSIONS}

The proposed filter bank allows the signal processing of the noise object with unknown length. The filter banks response for all TBD filters gives very good results (mean position errors about a few samples or pixel). The size of the object is rather small. The Monte Carlo test allows the estimation of the performance of TBD filters.

The main problem of ST TBD is the computation cost, but FPGA and GPGPUs devices allow the processing in real time [14]. The cost of the bank filter is linear and the number of the ST TBD blocks influences the results.

The application of specific implementation [15], [16], [17] for selected processing device gives the possibility of the more efficient implementation with lower computational cost, what is interesting research area.

\section{REFERENCES}

[1] S. Blackman and R. Popoli, Design and Analysis of Modern Tracking Systems. Artech House, 1999.

[2] M. Malanowski, "Algorithm for target tracking using passive radar," Intl. Journal of Electronics and Telecommunications, vol. 58 (4), pp. 345350, 2012.

[3] P. Samczyński, M. Wilkowski, and K. Kulpa, "Trial results on bistatic passive radar using non-cooperative pulse radar as illuminator of opportunity," Intl. Journal of Electronics and Telecommunications, vol. 58 (2), pp. 171-176, 2012.

[4] Y. Bar-Shalom, Multitarget-Multisensor Tracking: Applications and Advances, vol. II. Artech House, 1992.

[5] S. Blackman, Multiple-Target Tracking with Radar Applications. Artech House, 1986.

[6] Y. Boers, F. Ehlers, W. Koch, T. Luginbuhl, L. Stone, and R. Streit, "Track before detect algorithm," EURASIP Journal on Advances in Signal Processing, 2008.

[7] L. Stone, C. Barlow, and T. Corwin, Bayesian Multiple Target Tracking. Artech House, 1999.

[8] P. Mazurek, "Optimization of Bayesian track-before-detect algorithms for GPGPUs implementations," Electrical Review, vol. 86 (7), pp. 187 189, 2010.

[9] — , "Hierarchical track-before-detect algorithm for tracking of amplitude modulated signals," Advances in Intelligent and Soft Computing, vol. 102 - Image Processing and Communications Challenges 3, pp. 511-518, 2011.

[10] - "Track-before-detect algorithm for noise objects," Measurement Automation and Monitoring, vol. 56 (10), pp. 1183-1185, 2010.

[11] - "Comparison of different measurement spaces for spatio-temporal recurrent track-before-detect algorithm," Advances in Intelligent and Soft Computing, vol. 102 - Image Processing and Communications Challenges 3, pp. 157-164, 2011.

[12] _ "Chi-square statistic for noise object tracking in track-beforedetect systems," Poznań University of Technology Academic Journals - Electrical Engineering, vol. 71, pp. 177-184, 2012.

[13] _ "Application of dot product for track-before-detect tracking of noise objects," Poznań University of Technology Academic Journals Electrical Engineering, vol. 76, pp. 101-107, 2013.

[14] _ " "Code reordering using local random extraction and insertion (LREI) operator for GPGPU-based track-before-detect systems," Soft Computing, vol. 18 (6), pp. 1095-1106, 2013.

[15] — - "Estimation track-before-detect motion capture systems state space spatial component," Lecture Notes in Computer Science, vol. 4673 (Computer Analysis of Images and Patterns), pp. 149-156, 2007.

[16] _ , "Estimation of state-space spatial component for cuboid trackbefore-detect motion capture systems," Lecture Notes in Computer Science, vol. 5337 (Computer Vision and Graphics International Conference ICCVG 2008), pp. 451-460, 2009.

[17] M. John, M. Inggs, and D. Petri, "Real time processing of networked passive coherent location radar system," Intl. Journal of Electronics and Telecommunications, vol. 57 (3), pp. 363-368, 2011. 